ARIZA, Libardo; ITURRALDE, Manuel. "Mujer, crimen y castigo penitenciario". Polít. crim. Vol. 12, No 24 (Diciembre 2017), Art. 3, pp. 731-753.

[http://www.politicacriminal.cl/Vol_12/n_24/Vol12N24A3.pdf]

\title{
Mujer, crimen y castigo penitenciario
}

\section{Women, crime and prison}

\author{
Libardo Ariza ${ }^{1}$ \\ Dr. en Derecho, Universidad de Deusto \\ Profesor Asociado \\ Facultad de Derecho \\ Universidad de los Andes \\ lj.ariza20@uniandes.edu.co \\ Manuel Iturralde \\ Dr. en Derecho, London School of Economics \\ Profesor Asociado \\ Facultad de Derecho \\ Universidad de los Andes \\ miturral@uniandes.edu.co
}

\begin{abstract}
"Nevertheless a comparison of the criminal skull with the skulls of normal women reveals the fact that female criminals approximate more to males, both criminal and normal, than to normal women, especially in the superciliary arches in the seam of the sutures, in the lower jaw-bones, and in peculiarities of the occipital region. They nearly resemble normal women in their cheek-bones, in the prominence of the crotaphitic line, and in the median occipital fossa". 2
\end{abstract}

\section{Resumen}

El estudio de las relaciones entre mujer, crimen y formas de castigo ha sido uno de los asuntos más intensamente discutidos en la teoría criminológica y la política pública. Las variaciones en las aproximaciones, tanto a la explicación del crimen femenino, como a las respuestas institucionales concretas al mismo, han dado lugar a arreglos discursivos y prácticos concretos que moldean la comprensión pública sobre el conflicto de las mujeres con el derecho penal. La actual discusión en torno a la excesiva representación de las mujeres en el sistema de prisiones por delitos menores de drogas, así como la situación de la mujer presa madre, y la necesidad de considerar medidas alternativas al encarcelamiento, son algunos ejemplos de lo anterior. Este artículo pretende mostrar los retos analíticos y

\footnotetext{
${ }^{1}$ Este artículo es producto de la investigación "Análisis sociojurídico de la situación de las mujeres presas en Colombia", realizada entre febrero de 2012 y abril de 2013 en el establecimiento de reclusión para mujeres El Buen Pastor de Bogotá. La investigación fue financiada por el Centro de Investigaciones Sociojurídicas CIJUS, de la Facultad de Derecho de la Universidad de los Andes.

${ }^{2}$ LOMBROSO, Cesar; FERRERO, William, The Female Offender, New York: D. Appleton and company, 1898, p. 28.
} 
ARIZA, Libardo; ITURRALDE, Manuel. "Mujer, crimen y castigo penitenciario".

políticos que deben asumirse cuando se trata de encontrar respuesta a la compleja relación entre mujeres, delito y prisión.

Palabras clave: Mujeres, criminología, encarcelamiento, política criminal.

\begin{abstract}
The complex relations between women, crime and punishment had been intensively discussed both in criminology theory and public policy. Different transformations and variations in the assessment, explanation and responses to these issues, have shaped public understanding and criminal policy responses to women's social control through specific discourses on the nature of women deviance. Current discussions on women's over representation in the penal system for drug related crimes, as well as the need for specific responses to the intersection between incarceration and motherhood, are significant examples of the situation. In this article we aim to address the analytic and political challenges that should be considered in finding answers to women's crime and incarceration.
\end{abstract}

Key words: Women, imprisonment, criminology, criminal policy

\title{
Introducción
}

Según las estadísticas oficiales, pocas mujeres cometen delitos y aún menos van a prisión. En 2015 había alrededor de 700.000 mujeres recluidas alrededor del mundo (una tercera parte en los Estados Unidos). En el $80 \%$ de los sistemas penitenciarios y carcelarios las mujeres representan entre el 2 y el $9 \%$ de total de la población, aunque en el continente americano constituyen el $15 \%{ }^{3}$. ¿Cuál es entonces la relevancia de estudiar a fondo un fenómeno que los discursos penales y criminológicos imperantes parecen considerar secundario dada la marginalidad de la población que involucra? Existen al menos tres razones: primero, el notable aumento de la población reclusa femenina en prácticamente todo el mundo, y especialmente en América Latina y Colombia, durante las últimas dos décadas. Desde el año 2000 la población reclusa femenina aumentó un $50 \%$ a nivel mundial; el mayor incremento se dio en el continente americano (con un $23 \%)^{4}$. Según un informe de la Comisión de Derechos Humanos de la ONU, la población reclusa femenina a nivel mundial aumentó alrededor del $159 \%^{5}$. En Colombia, dicha población aumentó un $329 \%$ entre 1990 y $2013^{6}$.

Segundo, porque las experiencias de las mujeres frente al sistema penal y el encarcelamiento son distintas que las de los hombres y por lo tanto no son asimilables. Dicha diferencia se manifiesta, por una parte, en la sensación por parte de las mujeres privadas de la libertad de que experimentan una forma más intensa y discriminatoria de

\footnotetext{
${ }^{3}$ WALMSLEY, Roy, International Center for Prison Studies: World Female Imprisonment List. $3^{\text {rd }}$ ed., London: World Prison Brief, 2015, p. 2.

${ }^{4}$ WALMSLEY, International Center, cit. nota $\mathrm{n}^{\circ} 3$, p. 2.

${ }^{5}$ CENTRO DE ESTUDIOS LEGALES Y SOCIALES -CELS- et al, Mujeres en prisión. Los alcances del castigo, $1^{\text {a }}$ ed., Buenos Aires: Siglo Veintiuno Editores, 2011, p. 10-11.

${ }^{6}$ Instituto Nacional Penitenciario y Carcelario INPEC. Estadísticas oficiales.
} 
castigo, que se traduce en condiciones más precarias de privación de la libertad. Lo anterior, en parte, debido a la poca importancia que se le ha prestado a su situación por parte de la política criminal penitenciaria. Si bien es difícil, como se verá a lo largo de este texto, aseverar que el encierro penitenciario es más duro, más estricto, o más intenso en el caso de las mujeres, sí es posible afirmar que en algunos aspectos, y desde el punto de vista de las mujeres encarceladas, su experiencia en el campo penitenciario es distinta a la del encierro masculino ${ }^{7}$.

Para citar tan solo un ejemplo, las necesidades en términos de atención en salud de las mujeres son distintas de las de los hombres y son atendidas de forma deficiente y, en varias ocasiones, sin consideración alguna frente a su dignidad; los casos de mujeres que han tenido que dar a luz esposadas son un ejemplo extremo, mas no excepcional, de cómo en la prisión se ve el cuerpo y actitud de la mujer como una amenaza para la seguridad ${ }^{8}{ }^{9}$.

Igualmente, la particularidad de las relaciones entre las mujeres y el campo penitenciario se observa no solo en el caso de aquellas que han sido privadas de la libertad sino en situación de la mujer que es vinculada al sistema en condición de visitante. Las extensas y extenuantes filas de mujeres que esperan entrar a los establecimientos para visitar a la inmensa población masculina reflejan de manera clara la forma en que los rigores del encierro se trasladan a las mujeres. Las requisas, vejatorias o no, la provisión de alimentos y ropa limpia para que el hombre interno pueda sortear la semana de encierro, así como la unificación de los trabajos productivos y reproductivos en el mundo exterior, son sola algunas de los efectos diferenciales que el sistema penitenciario y carcelario surte sobre las mujeres. La mujer no solo suple a través de su trabajo reproductivo las falencias de un sistema que colapsa frente al hacinamiento, sino que asume el deber de mitigar los efectos destructivos del encierro penitenciario sobre el núcleo familiar. Además, la privación de la libertad tiene un impacto fuerte en las mujeres que la padecen y en su círculo familiar, especialmente a sus hijos, aún más cuando son menores de edad. Esto sucede en distintos países, como se verá a lo largo de este texto. En Colombia, aunque los datos oficiales son muy incompletos, se estima que el $53 \%$ de las mujeres presas son madres cabeza de familia, y la mayoría de sus hijos tiene entre 4 y 10 años de edad ${ }^{10}$.

A las anteriores circunstancias que definen en gran medida la experiencia subjetiva de las mujeres en prisión, deben sumarse los rasgos que comparten con los hombres reclusos: la mayoría de mujeres privadas de la libertad tienden a ser jóvenes, en situación de marginalidad y pobreza, bajos niveles educativos y pocas posibilidades de acceso al

\footnotetext{
${ }^{7}$ Por ello, el asunto, en nuestra opinión, recae en establecer descriptivamente, con base en la visión de las personas privadas de la libertad, cuáles son los aspectos particulares que distinguen su experiencia penitenciaria de aquella de la población masculina general. Esta posición metodológica, que incluye a los distintos participantes dentro del campo penitenciario, es desarrollada por Newbold, et. al. (2014).

${ }^{8}$ CARLEN, Pat; WORRALL, Anne, Analysing Women's Imprisonment, Cullompton: Willan Publishing, 2004, P. 61.

${ }^{9}$ VAN GUNDY, Alana; BAUMANN-GRAU, Amy, Women, Incarceration, and Human Rights Violations: Feminist Criminology and Corrections, London: Ashgate, 2014, pp. 65-86

10 BRICEÑO, Marcela, Mujeres y prisión en Colombia: Análisis desde una perspectiva de Derechos Humanos y Género, Bogotá: Procuraduría Delegada en lo Preventivo para Derechos Humanos y Asuntos Étnicos-Grupo de Asuntos Penitenciarios y Carcelarios, 2006, p. 30.
} 
ARIZA, Libardo; ITURRALDE, Manuel. "Mujer, crimen y castigo penitenciario".

mercado laboral. Sin embargo, la discriminación de género y los roles sociales que les son impuestos a las mujeres, contribuyen a que factores como la pobreza y la exclusión las afecte de forma más frecuente e intensa que a los hombres ${ }^{11}$.

Tercero, porque el análisis del encarcelamiento de las mujeres es un aspecto fundamental para entender el fenómeno del encarcelamiento masivo, que es una característica cada vez más saliente de las sociedades contemporáneas. Los estudios sobre encarcelamiento masivo a nivel global se han concentrado en analizar las relaciones entre la expansión penitenciaria, las transformaciones en el sistema productivo y el neoliberalismo ${ }^{12}$, haciendo especial énfasis en la explicación de la presencia desproporcionada de minorías nacionales y grupos étnicos en el sistema penitenciario ${ }^{13}$. En este contexto, la perspectiva de género apenas aparece. Con todo, el interés sobre el impacto de este fenómeno en el caso particular de las mujeres, ha dado lugar a importantes estudios sobre la intersección entre la identidad racial y la identidad de género ${ }^{14}$. La creciente participación de mujeres extranjeras en las prisiones de Europa occidental muestra su especial vulnerabilidad frente a la criminalización de las migraciones laborales globales Sur-Norte ${ }^{15}{ }_{-}{ }^{16}{ }^{17}$. La actual preocupación por la participación desproporcional de las mujeres en el sistema penitenciario por delitos relacionados con drogas es otro aspecto de lo anterior ${ }^{18}$.

Este texto pretende analizar críticamente los principales análisis y estudios que se han producido, particularmente en los últimos 30 años, sobre las mujeres privadas de la libertad. Lo anterior, con el propósito de mostrar las transformaciones que ha experimentado el discurso criminológico sobre la mujer, el crimen y el castigo penitenciario, para avanzar en la construcción de una agenda analítica y política que permitan encontrar rumbos alternativos en la definición de la respuesta institucional a la mujer en conflicto con el derecho penal. Para ello se discutirá cómo las perspectivas crítica, progresista y feminista, tanto del discurso penal como del criminológico, desde distintos puntos de vista y con distintos métodos de investigación, han coincidido en ciertos aspectos básicos: la supuesta marginalidad de las mujeres en prisión ha servido como forma de invisibilización de sus penosas condiciones de vida y de los efectos particularmente intensos que el encierro penitenciario surte sobre las mujeres y sus familias.

\footnotetext{
${ }^{11}$ CARLEN/WORRAN, Analysing Women's, cit. nota n ${ }^{\circ}$ 8, p. 93.

${ }^{12}$ DE GIORGI, Alessandro, "Immigration control, post-Fordism, and less eligibility: A materialist critique of the criminalization of immigration across Europe", Punishment \& Society, Vol. 12, N² (2010), pp. 147-167.

${ }^{13}$ WACQUANT, Löicq, "Suitable enemies: Foreigners and immigrants in the prisons of Europe", Punishment \& Society, vol. 1, N² (1999), pp. 215-222.

${ }^{14}$ Por ejemplo, ver SUDBURY, Julia, Global lockdown: Race, Gender, and the Prison Industrial Complex, New York: Routledge, 2005.

${ }^{15}$ MELOSSI, Dario, "In a peaceful life: Migration and the crime of modernity in Europe", Punishment \& Society, Vol. 5, N 4 (2003), pp. 371-397.

16 Por ejemplo, ver DAUVERGNE, Catherine, Making People Illegal: What Globalization Means for Migration and Law, Cambridge: Cambridge University Press, 2008.

${ }^{17}$ Por ejemplo, ver BARBERO, Iker, Las transformaciones del Estado y del Derecho ante el control de la inmigración, Bilbao: Ikuspegi, 2010.

18 ARIZA, Libardo; ITURRALDE, Manuel, "En contra de los pobres: justicia penal y prisiones en América Latina. El caso colombiano", en: GARGARELLA, Roberto (Ed.), El castigo penal en sociedades desiguales, Buenos Aires: Miño y Dávila, 2012, pp. 15-44.
} 
Como mostramos en este texto, dicha invisibilización también ha servido para legitimar un discurso particularmente punitivo frente a las mujeres etiquetadas como criminales, quienes son vistas como doblemente desviadas: son malas ciudadanas y malas mujeres. Así, además de actuar en contra de las normas jurídicas que regulan la convivencia social, la mujer criminal quebranta la norma natural que ordena su posición en el hogar, en el cuidado de la familia, y, en general, en las labores reproductivas. Este doble quebrantamiento, esta desviación dual, en virtud de la cual la mujer criminal desafía tanto la norma jurídica como la norma natural, hacen que el estigma que recae sobre ellas sea especialmente destructivo. Creemos que es en este contexto que deben ser interpretadas las tecnologías disciplinarias propias de la prisión, incluyéndolas dentro del arreglo más amplio de formas intensas de control social que una sociedad patriarcal ejerce, desde distintos ámbitos sociales y culturales, sobre las mujeres. Esto no quiere decir que la prisión sea la más importante, pero tampoco que es el dispositivo menos relevante. En efecto, y como se verá en el desarrollo de este texto, la experiencia penitenciaria, así como los programas específicos de tratamiento y rehabilitación, se basan en una idea particular de lo femenino. Por una parte, al intensificar un ideal de lo femenino basado en el cuidado del cuerpo, el paso por prisión reconstruye la delicadeza femenina y la aparta del recurso de la fuerza y la violencia. La prisión, en este sentido, desempodera. Mientras los hombres alzan pesas en la prisión, las mujeres organizan reinados de belleza y se reúnen en peluquerías, preparando su rostro y cuerpo para recibir a su visitante con la espera de no ser abandonada. Por otra parte, a través de la maternalización de la experiencia penitenciaria. La preeminencia, política y académica, del discurso de la maternidad, la familia y el cuidado, para hacer inteligible el encierro penitenciario femenino restringe el fenómeno a una de sus posibles múltiples facetas. Para que sea relevante, la mujer presa debe articular su experiencia en el crimen y castigo a través del lenguaje de la maternidad: intentar convertirse en la buena mujer madre que traicionó a través de su acto criminal.

\title{
1. Género y prisiones: malas ciudadanas y malas mujeres
}

\section{Masculinas e invisibles}

\begin{abstract}
"Criminology remains one of the most thoroughly masculinized of all social science fields"19

"In woman, also, this tendency to prevail by passive means rather than by assault is natural; and especially under a system of male control, where self-realization is secured either through the manipulation of man or not at all, a resort to trickery, indirection, and hypocrisy is not to be wondered at" 20
\end{abstract}

El discurso y las prácticas penales frente a las mujeres tradicionalmente han partido de una contradicción que tiene profundas raíces sociales y culturales: la concepción de que las

\footnotetext{
${ }^{19}$ BRITTON, Dana, "Feminism in Criminology: Engendering the Outlaw", Annals of the American Academy of Political and Social Science, Vol. 571, $\mathrm{N}^{\circ} 1$ (2000), pp. 57-76.

${ }^{20}$ THOMAS, W. I., "The Adventitious Character of Woman", American Journal of Sociology, Vol. 12, № 1(1906), pp. 32-44.
} 
ARIZA, Libardo; ITURRALDE, Manuel. "Mujer, crimen y castigo penitenciario”.

mujeres criminales son y no son mujeres al mismo tiempo ${ }^{21}{ }_{-}^{22}$. En efecto, desde el texto seminal de Lombroso y Ferrero ${ }^{23}$ sobre la mujer delincuente, en el cual sostienen que la mujer criminal se acerca más al hombre, criminal o no, que a la mujer normal, la duda sobre la identidad de la mujer que delinque va de la mano del castigo que recibe. Esto porque históricamente los regímenes penitenciarios, pensados y diseñados para hombres, han tratado a las mujeres como si fueran prisioneros (hombres); sin embargo, al mismo tiempo, los regímenes penitenciaros a los que son sometidas las mujeres suelen ser más severos, represivos y discriminatorios al basarse en ideologías y concepciones sobre la feminidad que tienden a prevalecer en la sociedad. Los estereotipos culturales y sociales dominantes representan a las mujeres criminales como perversiones de la naturaleza femenina.

Según Carlen y Worrall, tales estereotipos tradicionalmente se han basado en tres concepciones básicas: primero, la noción de que la conducta criminal es ante todo una actividad propia de los hombres, por lo que las mujeres que cometen delitos son doblemente desviadas: violan la ley penal y su feminidad; son malas ciudadanas y malas mujeres. Segundo, la tendencia a "patologizar" y "medicalizar" a las mujeres privadas de la libertad, quienes suelen ser vistas como menos robustas, física y sobre todo mentalmente, que los hombres. Tercero, una ansiedad social frente al papel de las mujeres en la familia y la sociedad, y la creencia de que las prisiones, y otras instituciones de control social (como los manicomios, los reformatorios, lo conventos y la familia misma), a través de sus reglas, regímenes y programas, pueden limitar los daños y sufrimientos padecidos por las mujeres, sus familias y la sociedad en general, una vez sean liberadas ${ }^{24}$.

Como lo señala Smart ${ }^{25}$, los mitos acerca de la maldad "inherente" de la mujer o su falta de inteligencia y habilidad, así como la creencia en su pasividad natural, informan los estudios clásicos sobre la criminalidad femenina. Esta suerte de determinismo biológico, sostiene Smart, no toma en cuenta los factores dominantes utilizados para dar cuenta del comportamiento delictivo de los hombres, como la clase, el status, poder y cultura. El resultado es un cuerpo de conocimiento que perpetúa estereotipos sobre la naturaleza de lo femenino, de tal manera que aquellas mujeres que cometen crímenes son catalogadas como criminales por naturaleza o en términos patológicos, al apartarse de la auténtica naturaleza biológicamente determinada de lo femenino.

Así, los regímenes de control social a los que son sometidas las mujeres desviadas (de su rol femenino), en los que las prisiones juegan un lugar cada vez más importante, han sido delineados por una combinación y superposición de ideologías sobre la feminidad (la mujer por naturaleza es maternal, tierna, sumisa, doméstica) que retratan a las mujeres delincuentes como masculinas, dementes y pervertidas; todo lo cual las aleja de su rol

\footnotetext{
${ }^{21}$ CARLEN/WORRAN, Analysing Women's, cit. nota n ${ }^{\circ} 8$, p. 1.

${ }^{22}$ Ver WORRALL, Anne, Offending women: Female Lawbreakers and the Criminal Justice System, London: Routledge, 1990.

${ }^{23}$ LOMBROSO/FERRERO, The Female Offender, cit. nota n ${ }^{\circ}$, p. 1.

${ }^{24}$ LOMBROSO/FERRERO, The Female Offender, cit. nota $\mathrm{n}^{\circ}$ 2, p. 2.

${ }^{25}$ SMART, Carol, “The New Female Criminal: Reality or Myth?”, British Journal of Criminology, Vol. 19, $\mathrm{N}^{\circ} 1$ (1979), pp. 50-59.
} 
tradicional en la familia, el mercado laboral y la sociedad ${ }^{26}$. Así, las mujeres privadas de la libertad no solo deben sufrir los rigores a los que son sometidos los hombres y que son propios del régimen carcelario, sino que adicionalmente deben soportar psicológica y físicamente el disciplinamiento a través de procesos impuestos de feminización, domesticación y medicalización ${ }^{27}{ }_{-}{ }_{-}{ }^{29}$.

Las ambiguas prácticas y discursos penales y penitenciarios sobre la mujer, que a la vez la invisibilizan y la tratan con mayor rigor, se han ido consolidando desde el siglo XIX a través del discurso penal moderno. Éste, basado en una perspectiva humanista y resocializadora, estableció la necesidad de separar espacialmente a las mujeres de los hombres recluidos para garantizar su seguridad (especialmente frente a formas de violencia física y sexual), pero sometiéndolas a espacios y tratamientos diseñados para los hombres, principal foco de atención del discurso y las prácticas penales y penitenciarias. No obstante, como se vio anteriormente, tales prácticas y discursos a su vez han sido atravesados por un discurso e ideología patriarcal sobre las mujeres que, al sobreponerse al discurso oficial del campo penal, ha terminado por tratarlas con mayor dureza y rigor.

Este desarrollo se debió en buena medida a las visiones patriarcales que se han tenido sobre las mujeres y que han estado presentes en la prisión desde sus comienzos. La concepción de las mujeres delincuentes como pervertidas, desnaturalizadas, malas madres, débiles mentales, así como la tendencia a infantilizarlas, dio lugar a que se ejerciera una vigilancia mucho más estrecha sobre ellas que sobre los hombres (de forma común a través de su medicalización); a que se les asignaran trabajos y formas de disciplina basadas en presupuestos, y prejuicios, sobre sus habilidades y limitaciones naturales ${ }^{30}$. Uno de los argumentos centrales de esta perspectiva sostiene que en las sociedades industriales existe una estratificación sexual del control social de los menores basada en la familia. Esta teoría sostiene que dicha estratificación se impone a través de una relación instrumental objetiva en la cual las madres, más que los padres, se encargan del control social de las hijas. Por ello, se sostiene que las hijas sean menos inclinadas que los hijos a tomar riesgos y, por lo tanto, que estén menos inclinadas a involucrarse en conductas delincuenciales ${ }^{31}$.

Tales tecnologías disciplinarias buscan hacer de malas mujeres buenas madres, hijas, esposas, amas de casa; dóciles y amorosas; dispuestas y preparadas para atender al hombre (bien sea su padre o esposo) y a sus hijos ${ }^{32}{ }_{-}^{33}$. Aquellas mujeres que rechazan dicho rol, y las formas de disciplinamiento que lo imponen, son vistas como peligrosas, agresivas,

\footnotetext{
${ }^{26}$ SMART, "The New Female", cit. nota ${ }^{\circ} 25$.

${ }^{27}$ Ver SMITH, Ann, Women in Prison: A Study in Penal Methods, London: Stevens, 1962.

${ }^{28}$ CARLEN, Pat, Women's Imprisonment: A Study in Social Control, London: Routledge \& K. Paul, 1983, p. 248.

${ }_{29}$ Ver DOBASH, Russell; DOBASH, R. Emerson; GUTTERIDGE, Sue, The Imprisonment of Women, Oxford: Blackwell, 1986.

${ }^{30}$ DOBASH/DOBASH/GUTTERIDGE, The imprisonment, cit. nota $\mathrm{n}^{\circ} 29, \mathrm{p} .61$.

${ }^{31}$ HAGAN, John; SIMPSON, John; GILLIS, A.R, "Feminist Scholarship, Relational and Instrumental Control, and a Power-Control Theory of Gender and Delinquency", The British Journal of Sociology, Vol. 39, $\mathrm{N}^{\circ} 3$ (1988), pp. 301-336.

${ }^{32}$ Ver SMITH, Women in Prison, cit. nota $\mathrm{n}^{\circ} 27$.

${ }^{33}$ CARLEN/WORRAN, Analysing Women's, cit. nota n ${ }^{\circ} 8$, p. 11.
} 
ARIZA, Libardo; ITURRALDE, Manuel. "Mujer, crimen y castigo penitenciario”.

indisciplinadas, histéricas, incapaces mentales, que por lo tanto requieren de tratamientos más severos: castigos que las hagan dóciles, aislamiento frente a las otras internas, tratamiento psiquiátrico basado en el uso de medicamentos que las tranquilice. De lo anterior se puede concluir que la invisibilización de la mujer desviada y de la mujer reclusa que trajo consigo el discurso y las prácticas penales y penitenciarias propias de la modernidad es engañosa. El bajo número de mujeres reclusas, en términos absolutos y en especial en comparación con los hombres, ha servido de justificación para su marginalización de las prácticas y los discursos penales y criminológicos. Pero esta marginalización oculta el hecho de que, si bien las mujeres parecen una fracción insignificante dentro de la población carcelaria, esto se puede deber en buena medida a que a su vez han sido sometidas a formas variadas e intensas de un control social patriarcal, a través de instituciones como la familia, la religión, el trabajo y la educación, así como de ideologías, estructuras y procesos de control relativos a la familia y al género que son cualitativamente distintos de aquéllos que se ejercen sobre los hombres ${ }^{34}{ }^{35}$. El énfasis en el estudio de las agencias e instituciones formales de control social, como la policía, el sistema judicial, las prisiones, dejó de lado importancia de los mecanismos de control informal, cuyas desviaciones no se verían representadas en las cifras oficiales dominantes que informan los estudios académicos principales. Así, se sostiene que dos imágenes bien establecidas desde el punto de vista cuantitativo -la exclusión de la mujer de la competencia por los resultados de la estratificación social y los menores índices de criminalidad de las mujeres- tienen como fuente común patrones de control social informal, los cuales son establecidos y perpetuados dentro del núcleo familiar ${ }^{36}$. La mujer no es controlada por el sistema de justicia criminal sino por su propia familia.

En este sentido, el denominado desvanecimiento de la mujer en el campo criminal debe relacionarse con los cambios significativos en los roles asignados a la mujer en la economía, la familia y la sociedad general. Así, tal desvanecimiento puede reflejar un giro hacia formas más privadas de control social impulsadas por las actitudes sociales cambiantes y la emergencia de la sociedad industrial ${ }^{37}$. En este sentido, la prisión, parafraseando a Foucault, es tan solo parte del vasto archipiélago carcelario destinado a controlar a las mujeres. De hecho, varios autores sostienen que si con respecto a los hombres menos mujeres cometen delitos y van a prisión, esto sucede en buena medida porque ellas están sometidas a muchas más formas de control social, como los discursos sobre su feminidad (que las consideran resignadas y protectoras, frágiles y dependientes), sus responsabilidades familiares, los contradictorios mensajes sobre su papel en la economía (la tensión entre hacer parte del mercado laboral y ser buena ama de casa, buena esposa y buena madre), la violencia implícita y explícita, física y sexual, a la que son sometidas por los hombres ${ }^{38} 39$.

\footnotetext{
${ }^{34}$ CARLEN/WORRAN, Analysing Women's, cit. nota n ${ }^{\circ}$ 8, p. 12.

${ }^{35}$ CARLEN, Pat (Ed.), Criminal Women, Cambridge: Polity Press, 1995.

${ }^{36}$ HAGAN, John; SIMPSON, John; GILLIS, A.R., "The Sexual Stratification of Social Control: A GenderBased Perspective on Crime and Delinquency", The British Journal of Sociology, Vol. 30, N 1 (1979), pp. 25-38.

${ }^{37}$ FEELEY, Malcolm; LITTLE, Deborah, "The vanishing female: The decline of women in the criminal process", 1687-1912", Law and Society Review, Vol. 25, N 4 (1991), pp. 719-757.

${ }^{38}$ Ver CARLEN, Criminal Women, cit. nota ${ }^{\circ} 35$.

${ }^{39}$ CARLEN/WORRAN, Analysing Women's, cit. nota n ${ }^{\circ}$ 8, p. 122.
} 
Como indica Carol Smart ${ }^{40}$, la criminología positivista (y androcéntrica) ha construido dos tipos de argumentos, biológicos y de socialización, para explicar la limitada presencia de mujeres ante cortes penales y en las prisiones: de una parte, varias teorías criminológicas argumentan que las mujeres son genéticamente menos inclinadas a desplegar comportamientos agresivos, y por lo tanto a delinquir, pues por naturaleza son protectoras y proveedoras.

De otra parte, las mujeres son socializadas para conformarse a las normas sociales, por medio de la interiorización de las expectativas que genera su rol de género; desde pequeñas se les enseña lo que significa ser mujer en una determinada sociedad y la conducta que en consecuencia se espera de ellas. Esto hace que las mujeres presenten una menor tendencia a meterse en problemas con la ley. Además, cuando llegan a la edad adulta, las mujeres tienen menos oportunidades de cometer delitos pues deben ocuparse de sus hogares y sus hijos. Si no logran lidiar con estas responsabilidades, lo más común es que busquen ayuda médica o psiquiátrica, en lugar de incurrir en conductas desviadas.

La aparente invisibilización de las mujeres reclusas y su carácter de doblemente desviadas han propiciado un tratamiento y un control disciplinarios mucho más rigurosos y discriminatorios frente a las mujeres, que además son desproporcionados frente a la gravedad de las conductas cometidas y el riesgo social que representan. Si el espacio carcelario es una zona gris propicia para la suspensión del Estado de derecho y el despliegue de tecnologías disciplinarias, lo es aún más en el caso de las mujeres, sometidas al discurso unidimensional sobre lo femenino que las etiqueta como doblemente desviadas y necesitadas de una mano firme, como si de niñas o incapaces mentales se tratara, para que recobren su feminidad. En este aspecto fundamental se centra su tratamiento penitenciario; ya es un lugar común constatar que los programas de rehabilitación en las prisiones de mujeres suelen apuntar al desarrollo de habilidades y actitudes que se consideran propias de

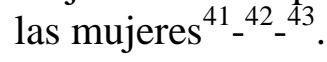

\section{La perspectiva feminista en un contexto punitivo}

A pesar de que las anteriores críticas al régimen de reclusión de las mujeres hoy parecen obvias, hasta antes de los ochenta la criminología y el discurso penal eran dominados por una visión masculina que ignoraba la perspectiva de género. Incluso posturas progresistas no eran sensibles frente la situación de las mujeres en prisión y lo que implicaba. Pero desde los ochenta, impulsados por la vigorosa corriente feminista, los estudios sobre mujeres que cometen crímenes y que son encarceladas, son mucho más abundantes y críticos frente a las formas de control social que se ejercen sobre las mujeres, especialmente las que son etiquetadas como delincuentes, y que son distintas a las que se ejercen sobre los hombres. ${ }^{44}$

\footnotetext{
${ }^{40}$ SMART, Carol, "Law, Crime and Sexuality", Essays in Feminism, Vol. 1, London: Sage Publications, 1995 , p. 40 y ss.

${ }^{41}$ CARLEN, Women's Imprisonment, cit. nota $\mathrm{n}^{\circ} 28$, pp. 66-73.

${ }^{42}$ Ver DOBASH/DOBASH/GUTTERIDGE, The imprisonment, cit. nota $\mathrm{n}^{\circ} 29$.

${ }^{43}$ DEVLIN, A, Invisible Women. What's Wrong with Women's Prisons?, Winchester: Waterside Press, 1998.

${ }^{44}$ CARLEN/WORRAN, Analysing Women's, cit. nota n ${ }^{\circ} 8$, p. 108.
} 
ARIZA, Libardo; ITURRALDE, Manuel. "Mujer, crimen y castigo penitenciario".

Carlen y Worrall ${ }^{45}$ señalan que este tipo de estudios hizo cinco grandes contribuciones al entendimiento de los significados del encarcelamiento de las mujeres. Primero, que los crímenes cometidos por mujeres ocurren en circunstancias distintas de aquéllos cometidos por hombres, que denotan formas más profundas de discriminación y opresión, que incluyen aspectos como la raza y la clase social. Segundo, que el perfil de las mujeres encarceladas es distinto del de su contraparte masculina; por ejemplo, menos mujeres que hombres tienden a tener algún tipo de empleo u oficio antes de ser encarceladas; muchas de ellas han sufrido distintas formas de violencia física y sexual antes de ir a prisión ${ }^{46}$. Así, la criminología feminista debe tener en cuenta no solo el género, sino también cuestiones de clase, raza e imperialismo para dar cuenta de por qué la mayoría de las mujeres encarceladas han cometido crímenes menores, por qué un número desproporcionado de ellas pertenece a minorías étnicas y por qué la mayoría han vivido en condiciones de pobreza alguna vez en sus vidas ${ }^{47}$.

Tercero, que las respuestas penales frente a las conductas desviadas de las mujeres se construyen a partir de estereotipos de la mujer y la feminidad que contribuyen a su opresión ${ }^{48}$. Cuarto, a través de sus investigaciones, varias feministas se han preguntado si la criminología feminista es un proyecto viable y deseable y si las 'mujeres' desviadas deben ser un foco de atención del feminismo ${ }^{49}$. Quinto, y de forma opuesta a las feministas escépticas, varias investigadoras sociales han combinado su trabajo académico con el activismo político para transformar la situación de opresión de las mujeres que deben enfrentarse al sistema penal y carcelario ${ }^{50}$. De esta forma, la perspectiva feminista frente a la criminología y el discurso penal, lejos de ser una corriente monolítica, se ha caracterizado por los fuertes debates y desacuerdos internos, no solo sobre cómo debería proceder, sino sobre si debería hacerlo desde estas disciplinas, estructuradas desde una perspectiva masculina y excluyente ${ }^{51}$.

\footnotetext{
${ }^{45}$ CARLEN/WORRAN, Analysing Women's, cit. nota n ${ }^{\circ}$ 8, p. 109.

${ }^{46}$ En el caso colombiano, según un estudio publicado en la Revista Criminalidad, de la Policía Nacional (basado en una muestra de 135 encuestas a mujeres en 4 centros de reclusión distintos, que representan el $10 \%$ de la población condenada) revela que más del $53 \%$ de las mujeres encuestadas manifiestan provenir de hogares donde fueron sometidas a diversas formas de maltratos y abusos. El 78\% declaró haber sido víctima de abusos (físicos o sexuales), donde los perpetradores fueron la pareja (61,7\% de os casos), algún miembro de la familia (50\%) y desconocidos y/o autoridades (29,5\%) (NORZA-CÉSPEDES, Ervyn; GONZÁLEZROJAS, Andrea; MOSCOSO-ROJAS, Manuel; GONZÁLEZ-RAMÍREZ, Juan David, "Descripción de la criminalidad femenina en Colombia: factores de riesgo y motivación criminal", Revista Criminalidad, Vol. 58, $\mathrm{N}^{\circ} 1$ (2012), pp. 339-357).

${ }^{47}$ Ver CARLEN, Pat, Criminal women and criminal justice: The limits to, and potential of, left realist perspectives, London: Sage, 1992.

${ }^{48}$ CARLEN, Pat, Sledgehammer: Women's Imprisonment at the Millennium, London: Palgrave Macmillan, 1998, pp. 1-46.

${ }^{49}$ Ver CARLEN, Criminal Women, cit. $\mathrm{n}^{\circ} 35$.

${ }^{50}$ Ver COUSINS, Mark, "Men's rea: a note on sexual difference, criminology and the law", en: CARLEN, Pat; COLLISON, Mike (Eds.), Radical Issues in Criminology, New Jersey: Barnes and Noble Books, 1980.

${ }^{51}$ CAIN, Maureen, "Feminists transgress criminology", Introducción en CAIN, Maureen (Ed.), Growing Up Good: policing the behavior of girls in Europe, London: Sage, 1989; SMART, Carol, "Feminist approaches to Criminology or Post modern woman meets atavistic man", en: GELSTHORPE, Lorraine; MORRIS, Allison (Eds.), Feminist Perspectives in Criminology, Buckingham: Open University Press, 1990. CARLEN, Criminal Women, cit. nota ${ }^{\circ} 35$.
} 
A pesar de que la perspectiva y la criminología feministas durante los últimos treinta años han visibilizado estas tecnologías y discursos disciplinarios, así como las relaciones de poder que ejercen los hombres sobre las mujeres, su impacto sobre la situación y la visión de las mujeres prisioneras han sido limitados, especialmente en América Latina. Esto se debe en buena medida a que, paradójicamente, durante ese periodo, las prácticas y el discurso punitivos que se han impuesto en buena parte del mundo, han relegitimado el uso de la prisión como principal forma de castigo y han dado lugar a un impresionante aumento de la población reclusa femenina, proporcionalmente mayor al de la población masculina. Aunque los discursos penal y penitenciario, como suelen hacerlo, se han adaptado a la crítica feminista, incluso adoptando parte de su retórica, siguen predominando las formas de control intensivo sobre las mujeres, bajo el manto de legitimidad que les proporcionan los nuevos discursos, pero bajo un contexto aún más punitivo.

La crisis del Estado bienestar en Europa y Estados Unidos (desde finales de los setenta) y el surgimiento de regímenes políticos y económicos conservadores, bajo la bandera del neoliberalismo, endurecieron las condiciones de vida de los jóvenes en situación de marginalidad. Los recortes en los programas de asistencia social y un enfoque mucho más punitivo para enfrentar situaciones de marginalización social, afectaron de forma desproporcionada a los jóvenes de la periferia urbana, y especialmente a las mujeres y minorías étnicas, a quienes les comenzó a resultar mucho más difícil beneficiarse de programas de acceso a la vivienda y el trabajo.

Las jóvenes madres que vivían solas con sus hijos comenzaron a ser señaladas como el ejemplo vívido del fracaso y los efectos perniciosos del Estado bienestar. La retórica conservadora de los gobiernos las mostraba como una amenaza para la institución familiar y el bienestar económico, al tiempo que su situación de desempleo y falta de educación, profundizaba su marginalidad. Así, la retórica y prácticas punitivas, tanto en el campo penal como en el de la asistencia social, se concentraron en la población joven y marginal, y particularmente en las madres solteras ${ }^{52}$.

Como indica Murray ${ }^{53}$, la retórica conservadora construía una narración según la cual los barrios en los que abundaban familias desestructuradas (generalmente por la ausencia del padre) y encabezadas por madres solteras, eran el caldo de cultivo de una subclase (underclass) de jóvenes (mal)criados por madres permisivas y un Estado bienestar cómplice, quienes eran reacios al trabajo honesto y en cambio se entregaban al consumo de alcohol y drogas, y a conductas antisociales, violentas y criminales. Este tipo de discurso afloró en diversas teorías criminológicas, como las teorías del control, que pretendían explicar, y exagerar, el desorden social reinante, con base en una teoría del crimen que lo entendía como el resultado de la falta de control, especialmente sobre los niños y jóvenes, que crecían en familias desestructuradas y bajo la tutela de padres ausentes y madres irresponsables, que eran vistas como la raíz de todo mal $^{54}$.

\footnotetext{
${ }^{52}$ CARLEN/WORRAN, Analysing Women's, cit. nota ${ }^{\circ} 8$, p. 13.

${ }^{53}$ Ver MURRAY, Charles, The Emerging British Underclass, London: Institute of Economic Affairs, 1994.

${ }^{54}$ KENNEDY, Helena, Eve was Framed: Women and British Justice, London: Chatto \& Windus, 1992, pp. 69-87.
} 
ARIZA, Libardo; ITURRALDE, Manuel. "Mujer, crimen y castigo penitenciario".

Como resultado de esta demonización de las jóvenes madres solteras, los gobiernos conservadores del Reino Unido y los Estados Unidos, durante los ochenta y los noventa, promulgaron legislaciones de corte punitivo que pretendían (a través de la restricción de la asistencia social y de mayores mecanismos de control para conceder la poca que quedaba) disuadir a las mujeres jóvenes y solteras, que no eran sostenidas por hombres, de tener hijos. En el campo penal, este punitivismo se manifestó en el aumento de condenas privativas de la libertad en contra de mujeres que reuniesen este perfil, en lugar de la aplicación de medidas alternativas y en detrimento de la acción de los servicios sociales del Estado $^{55}{ }_{-}^{56}$.

Aunque en el contexto latinoamericano no se puede hablar de un desmonte del Estado bienestar a favor del Estado neoliberal (al menos no de la forma en que sucedió en Estados Unidos y Europa), dadas las distintas trayectorias históricas de los países de la región, sí es posible afirmar que la imposición del modelo neoliberal en buena parte de la región ha tenido un fuerte impacto sobre el modelo de Estado y régimen político que rigen a las sociedades latinoamericanas. Parte esencial de este modelo es el despliegue de un Estado autoritario y punitivo, particularmente frente a las clases desposeídas, que son construidas discursivamente no como una población en riesgo, sino como personas que representan un riesgo para la sociedad y que por lo tanto deben ser tratadas a través de medios incapacitadores y punitivos ${ }^{57}{ }_{-}^{58}$. Las mujeres jóvenes, madres cabeza de familia y en situación de pobreza de la región, como en Europa y Estados Unidos, se han convertido en objetivo de dichas políticas punitivas.

Lo anterior explica, al menos parcialmente, el aumento de la población reclusa femenina durante las últimas dos décadas (no solo en el Reino Unido y los Estados Unidos, sino en diversas partes del mundo, entre ellas América Latina), y particularmente de jóvenes condenadas por delitos de narcotráfico, bien se trate de consumo o tráfico de estupefacientes ${ }^{59}{ }^{60}{ }_{-}{ }^{-}{ }^{62}$. Así, entre 1993 y 2001, la población femenina del Reino Unido

\footnotetext{
${ }^{55}$ WORRALL, Anne, “Out of place: Female offenders in court”, Probation Journal, Vol. 28, N 3 (1981), pp. 90-93.

${ }^{56}$ Ver CARLEN, Women's Imprisonment, cit. nota $n^{\circ} 28$.

${ }^{57}$ ITURRALDE, Manuel, La revolución desde arriba: la sociología política del neoliberalismo de Wacquant y su relevancia para América Latina, Bogotá: Siglo del Hombre, Universidad de los Andes, Pontificia Universidad Javeriana-Instituto Pensar, 2013. ITURRALDE, Manuel, "Prisiones y castigo en Colombia: la construcción de un orden social excluyente", en: ARIZA, Libardo; ITURRALDE, Manuel, Los muros de la infamia. Prisiones en Colombia y América Latina, Bogotá: Colección CIJUS-Uniandes, 2011. ITURRALDE, Manuel, Castigo, liberalismo autoritario y justicia penal de excepción, Bogotá: Siglo del Hombre, Universidad de los Andes, Pontificia Universidad Javeriana-Instituto Pensar, 2010. ITURRALDE, Manuel, "Democracies without Citizenship: Crime and Punishment in Latin America", New Criminal Law Review, Vol. 13, N² (2010), pp. 309-332.

${ }^{58}$ ARIZA/ITURRALDE, "En contra de los pobres", cit. nota $\mathrm{n}^{\circ} 18$.

${ }^{59}$ DENNIS, Norman; ERDOS, George, Families Without Fatherhood, London: IEA Health and Welfare Unit, 1992.

60 EDWARDS, Susan, Women on Trial. A Study of the Female Suspect, Defendant and Offender in the Criminal Law and Criminal Justice System, Manchester: Manchester University Press, 1984.

${ }^{61}$ EATON, Mary, Justice for Women? Familiy, Court and Social Control, Buckingham: Open University press, 1986.

${ }^{62}$ WORRALL, Offending women, cit. nota ${ }^{\circ} 22$.
} 
aumentó un $145 \%{ }^{63}$. En Colombia, la población reclusa femenina aumentó un 329\% entre 1990 y $2013^{64}$; en Estados Unidos aumentó un 26\% entre 2001 y 2009; la de España un 46\% entre 2000 y 2011; la de Francia un 31\%; la de Alemania un $10 \%$ entre 2000 y 2011; la de Brasil un 252\% entre 2000 y 2011; la de Chile un $87 \%$ entre 2003 y 2011; la de Uruguay un $99 \%$ entre 2006 y 2011; la de Argentina un 25\% entre 2005 y 2010; la de Venezuela un $156 \%$ entre 2001 y 2010; la de México un 39\% entre 2001 y 2011; la de El Salvador un $416 \%$ entre 2000 y 2010; la de Guatemala un 95\% entre 2005 y 2010; la de India un 69\% entre 2000 y 2009; la de China un 79\% entre 2000 y 2010; la de Japón un $60 \%$ entre 2000 y 2010; la de Australia un $46 \%$ entre 2000 y $2011^{65}$.

Como muestran las cifras anteriores, la población mundial femenina está creciendo en los cinco continentes $^{66}$; en promedio un $16 \%$ entre 2006 y $2012^{67}$. El mayor crecimiento se ha vivido en el continente americano, con un promedio del $23 \%$ en los últimos seis años ${ }^{68}$. El discurso penal y penitenciario predominante encuentra la causa de dicho aumento en la responsabilidad individual de las mujeres y deja de lado otros factores como las condiciones de exclusión y pobreza, el grado de victimización y otras formas de opresión de raza, de clase- a las que se ven enfrentadas las mujeres que terminan en prisión.

La criminología oficial y la retórica de los programas de tratamiento en las prisiones, se centran en la actitud de las prisioneras frente a su conducta criminal. Según éstas, el cambio en la forma de pensar de la mujer delincuente frente a su comportamiento reduciría las posibilidades de que vuelva a delinquir ${ }^{69}$. Adicionalmente, este discurso, apoyándose en el populismo punitivo ${ }^{70}$ que recorre buena parte de los campos penales contemporáneos, entiende la situación de marginalidad de las mujeres que cometen delitos menores, no como un indicativo de que requieren medidas distintas a la prisión, sino justamente lo contrario: su marginalidad es un indicador de posible reincidencia. La mejor forma de prevenir esto, sigue el discurso penal imperante, es por medio del encarcelamiento de dichas mujeres quienes además deben permanecer más tiempo en prisión. Por ejemplo, en Colombia, aunque las mujeres suelen tener penas más cortas que los hombres (lo cual es una tendencia global), su tiempo de reclusión ha tendido a aumentar en los últimos años.

Esta línea de pensamiento, y de tratamiento, desconoce por completo cómo los factores estructurales arriba mencionados también juegan un rol fundamental en las razones y motivos por los que las mujeres violan la ley penal. A su vez, este enfoque terapéutico, centrado en las actitudes y conducta de la mujer, aislada de su contexto, fortalece el discurso penal y criminológico que responsabiliza a las mujeres por las decisiones que

\footnotetext{
${ }^{63}$ CARLEN/WORRAN, Analysing Women's, cit. nota ${ }^{\circ} 8$, p. 15.

${ }^{64}$ INSTITUTO NACIONAL PENITENCIARIO Y CARCELARIO, 2013, Estadísticas oficiales, disponibles en:

http://www.inpec.gov.co/portal/page/portal/Inpec/Institucion/Estad\%EDsticas/Estadisticas/Estad\%EDsticas

${ }^{65}$ WALMSLEY, International Center, cit. nota $\mathrm{n}^{\circ} 3$.

${ }^{66}$ El International Centre for Prison Studies recopila la información disponible de 212 sistemas penales en países independientes y territorios dependientes (ICPS 20012:1).

${ }^{67}$ WALMSLEY, International Center, cit. nota $\mathrm{n}^{\circ} 3, \mathrm{p} .1$.

${ }^{68}$ WALMSLEY, International Center, cit. nota $\mathrm{n}^{\circ} 3, \mathrm{p} .1$.

${ }^{69}$ CARLEN/WORRAN, Analysing Women's, cit. nota ${ }^{\circ} 8$, p. 20.

${ }^{70}$ PRATT, John, Penal Populism, London: Routledge, 2007.
} 
ARIZA, Libardo; ITURRALDE, Manuel. "Mujer, crimen y castigo penitenciario".

tomaron y, por tal razón, las envían a prisión. Ésta relegitima su función al tratar a las internas como mujeres en estado de riesgo y que representan un riesgo, por lo que deben ser aisladas. Y solo dejarán de representar un riesgo, si se someten al tratamiento penitenciario, que las ayudará a cambiar de actitud frente a su forma de actuar y ver el mundo, en lugar de ayudarlas a enfrentar la precariedad de sus condiciones de vida fuera de prisión. Como señalan Carlen y Worrall $^{71}$, dichos programas terapéuticos y de responsabilización individual ignoran por completo que las mujeres ya saben cómo pensar sobre sus problemas. Su problema es poder actuar frente a él, o tener la oportunidad de hacerlo.

A partir de las críticas que el feminismo hace sobre el control social de las mujeres por medio de la prisión, así como de los discursos que lo legitiman, varias autoras feministas han propuesto una criminología y penología feministas, sensibles frente a las necesidades y particularidades de las mujeres. En este sentido, se debería buscar que la regulación penal de las mujeres sea un último recurso que no incremente aún más su opresión. Y, de manera concomitante, la regulación penal de los hombres no debe brutalizarlos y hacerlos aún más violentos y opresores de las mujeres ${ }^{72}$.

Especial énfasis debe hacerse en la regulación penal de las mujeres como último recurso. Dado que los estudios demuestran de forma constante que las mujeres suelen tener carreras criminales más cortas y menos serias que las de los hombres, así como menores tasas de reincidencia, no debería ser necesario aumentar el número de mujeres que van a prisión. La seguridad pública no se verá afectada por un mayor y más imaginativo uso de penas alternativas a la cárcel que además aborden los factores de justicia social (vivienda, ingreso, salud, educación y empleo) que afectan de forma desproporcionada a las mujeres que caen en las redes del sistema penal y penitenciario. Pero aparte de estos factores estructurales, las mujeres que han violado la ley penal deben recibir ayuda para sentir que son personas valiosas, que pueden apoyarse y brindar apoyo a partir de relaciones recíprocas y de muto respeto en lugar de relaciones subordinadas y de explotación ${ }^{73}$.

\section{La experiencia penitenciaria de las mujeres}

Como se mostró en las secciones anteriores, la literatura en esta materia tiende a coincidir en que el encarcelamiento femenino constituye una forma de continuar y profundizar la discriminación y el control social de las mujeres por parte de una sociedad patriarcal ${ }^{74}$. Más allá de los discursos criminológicos generales que intentan dar cuenta de la criminalidad femenina, la experiencia penitenciaria de la mujer supone identificar y analizar aquellos aspectos diferenciales que la caracterizan, así como su relación con la experiencia penitenciaria general masculina. En este sentido, la pregunta que se formula se refiere a si es distinta la experiencia penitenciaria de hombres y mujeres. Y, si es este el caso cuáles son esos factores diferenciales y cómo pueden ser identificados. En esta sección intentaremos esbozar brevemente las principales respuestas a las anteriores cuestiones, con

\footnotetext{
${ }^{71}$ CARLEN/WORRAN, Analysing Women's, cit. nota n ${ }^{\circ} 8$, p. 21.

${ }^{72}$ CARLEN, Pat, Alternatives to Women's Imprisonment, Oxford: Open University Press, 1990, p. 114.

${ }^{73}$ CARLEN/WORRAN, Analysing Women's, cit. nota ${ }^{\circ}$ 8, pp. 151-152.

${ }^{74}$ CARLEN/WORRAN, Analysing Women's, cit. nota ${ }^{\circ} 8$, pp. ix- x.
} 
especial énfasis en aquellos estudios que se ocupan de encontrarla en la experiencia subjetiva del sufrimiento penitenciario por parte de las mujeres privadas de la libertad.

Al igual que en la literatura criminológica, la aparición de la mujer en los estudios sobre el mundo del encierro es una cuestión relativamente reciente. En efecto, la experiencia de la mujer en prisión no se veía reflejada con suficiente intensidad en los estudios que abordan la cuestión del orden social interno penitenciario. Los relatos clásicos fundacionales sobre el mundo del encierro se desarrollaron en instituciones totales masculinas. La preocupación que impulsó este tipo de trabajos se concentraba en definir el sistema social imperante en los establecimientos de máxima seguridad ${ }^{75}$, indagando por la formación de un sistema social particular que buscaba alcanzar la difícil meta de la estabilidad. Instituciones cerradas, que realizan un reclamo de control casi total sobre la vida de hombres privados de la libertad contra su voluntad, en donde la violencia es la relación social dominante, constituían el escenario privilegiado de los estudios sobre la cultura penitenciaria. Los protagonistas de estos relatos eran hombres dominados por la institución total. El robusto cuerpo de conocimiento sobre la vida en prisión se erigió sobre la base del encierro masculino. El estudio de la "cultura de las personas presas" "76 y las descripciones de la "comunidad carcelaria",77, los análisis sobre la experiencia de la vida en prisión, así como los estudios sobre "prisionización" y mecanismos de adaptación al encierro, han tenido como objeto el fenómeno del encierro masculino ${ }^{78}$. En el ámbito local, en términos generales, sucede lo mismo ${ }^{79}$ con la excepción de algunos recuentos sobre las relaciones entre la criminalización de estupefacientes y su impacto en el encarcelamiento femenino.

Con todo, ciertos estudios han empezado a dirigir la mirada al interior de las prisiones de mujeres, mostrando que tomarse en serio la experiencia penitenciaria de las mujeres supone identificar y analizar aquellos aspectos diferenciales que la moldean -teniendo en cuenta su relación con la experiencia penitenciaria general- así como elucidar dichos factores diferenciales a través del discurso de las propias mujeres que se ven enfrentadas al castigo penitenciario. En este sentido, es posible distinguir dos aspectos en los cuales podrían hallarse los rasgos distintivos de la experiencia penitenciaria femenina: el diseño institucional penitenciario y la experiencia subjetiva del castigo intramural.

\section{Mujeres en cárceles de hombres}

La vulnerabilidad especial de las mujeres privadas de la libertad se observa en la infraestructura penitenciaria y en las condiciones concretas de reclusión. Por lo general, se

\footnotetext{
${ }^{75}$ Ver SYKES, Gresham M., The Society of Captives, Princeton: Princeton University Press, 1958.

${ }^{76}$ FINKELSTEIN, Ellis, Prison Culture: An Inside View, Averbury: Aldershot, 1993.

${ }^{77}$ CLEMMER, Donald, The Prison Community. Boston: Rinehart, 1958.

${ }^{78}$ GOFFMAN, Irvin, Asylums: Essays on the social situation of mental patients and other inmates, London: Penguin Books, 1961. ADAMS, Kenneth, “Adjusting to Prison Life”, en: TONRY, Michael (Ed.), Crime and Justice: A Review of Research, Chicago: University of Chicago Press, 1992, pp. 275-359. DE ROSIA, Victoria, Living inside prison walls: adjustment behavior, Connecticut: Praeger, 1998,

${ }^{79}$ ARIZA, Libardo, "Dados sin números: un acercamiento al orden social en la cárcel La Modelo", Revista de Derecho Público, No 26 (2011), pp. 1-21. CARRILlO, William, "From my Prison Cell. Time and Space in Prison in Colombia, an ethnographic approach", Latin American perspectives, Vol. 28, N 116 (2001), pp. 149-164.
} 
ARIZA, Libardo; ITURRALDE, Manuel. "Mujer, crimen y castigo penitenciario".

señala que la infraestructura penitenciaria ha sido diseñada para la reclusión de hombres, relegando a las mujeres a espacios menores que cuentan con acceso limitado a los programas institucionales donde se llevan a cabo las actividades propias del tratamiento penitenciario. Cuando se habilitan establecimientos especialmente dedicados a la reclusión de mujeres, espacialmente separados de los masculinos, estos sufren mayores tasas de hacinamiento y de peores condiciones habitacionales y de salubridad. Los escasos recursos asignados para la creación, adecuación y mantenimiento de los establecimientos penitenciarios femeninos plantean una cuestión adicional de distribución de los recursos del sistema penitenciario y carcelario, en la cual la adaptación y creación de una infraestructura especial para la reclusión de mujeres ocupa el último lugar en la agenda de prioridades de una política penitenciaria agobiada por la presión del hacinamiento masculino ${ }^{80}{ }^{81}$.

El carácter masculino de los establecimientos penitenciarios genera violaciones particulares de los derechos de las mujeres privadas de la libertad. Como lo resaltan Van Gundy y Baumann-Grau ${ }^{82}$, esto es especialmente significativo en el caso de los derechos reproductivos. En efecto, además de carecer de programas que garanticen embarazos y alumbramientos saludables, la dieta alimenticia no toma en cuenta las necesidades especiales de las mujeres en periodos de gestación y lactancia. Van Gundy y BaumannGrau, además señalan que dichas violaciones se incrementan por los riesgos que suponen los traslados a hospitales sin vehículos adecuados y por la ausencia de un cuidado prenatal deliberado como forma punitiva o como una negación que se desprende de la simple negligencia. La dureza del retorno al encierro tras el alumbramiento y la falta de atención a esta situación incrementarían el sufrimiento causado por la ausencia de programas especiales $^{83}$. Lo mismo ocurriría con la definición de los programas de atención pospenitenciaria, los cuales no tienen en cuenta que tras el paso por prisión las mujeres experimentan un riesgo mayor de mortalidad, como lo muestran las investigaciones que intentan elucidar las relaciones entre el trauma penitenciario y las experiencias posteriores de marginalización posteriores a la liberación. En este sentido, se señala que estas mujeres sufren un trauma acumulado que es el resultado de la violencia doméstica, el abuso sexual, la separación respecto de sus hijos y la intervención e institucionalización estatal ${ }^{84}$.

\footnotetext{
${ }^{80} \mathrm{El}$ discurso reformista y humanista que se impuso desde el siglo XIX pretendía que las prisiones, los sanatorios y reformatorios eran lugares ideales para domesticar y entrenar a las mujeres conflictivas, recalcitrantes o simplemente desafortunadas. Bajo esta premisa, dichos discursos sostenían que tales sitios de reclusión eran mucho más benignos que aquellos destinados para los hombres. Aunque en un principio se pudo constatar que los centros de reclusión de mujeres eran espacios y ambientes mucho menos hostiles y duros que los de los hombres, el paso del tiempo demostró que, de forma sutil pero penetrante, las técnicas de disciplinamiento impuestas a las mujeres y sus condiciones de reclusión eran desproporcionadamente más severas que aquéllas impuestas a los hombres, especialmente si se tiene en cuenta que la mayoría de mujeres privadas de la libertad no suelen cometer delitos violentos, no son reincidentes ni tienen una larga carrera criminal a cuestas, o al menos no en la misma proporción que los hombres.

${ }^{81}$ ALMEDA, Elizabet, "Women's imprisonment in Spain”, Punishment and Society, Vol. 7, N² (2005), pp. 183-199.

${ }^{82}$ VAN GUNDY/BAUMANN-GRAU, Women, Incarceration, cit. nota $\mathrm{n}^{\circ}$ 9, p. 65.

${ }^{83}$ OWEN, Barbara, In the mix: Struggle and Survival in a Women's prison, Albany: State University of New York Press, 1998, pp. 119-166.

${ }^{84}$ CARLTON, Bree; SEGRAVE, Marie, “Women's survival post-imprisonment: Connecting imprisonment with pains past and present”, Punishment \& Society, Vol. 13, N 5 (2011), pp. 551-570.
} 
El diseño de los programas de tratamiento penitenciario se encuentra lastrado por las mismas deficiencias pues responden, por lo general, a aquel que se ha hecho para los establecimientos masculinos y, cuando intentan ajustarse al caso particular de la reclusión de mujeres, hacen énfasis en el desarrollo de ciertas actividades que se basan en una noción problemática de lo femenino. La elaboración de artesanías, adornos y abalorios como la actividad diaria de la mujer privada de la libertad, contrasta con los talleres masculinos en los cuales los internos hombres desarrollan actividades propias de la formación de un obrero asalariado, desde carpintería hasta cursos de metalmecánica.

\section{El sufrimiento penitenciario}

Las deficiencias institucionales generales que se acaban de mencionar hacen de las mujeres uno de los grupos más vulnerables al interior de las prisiones. Las mujeres privadas de la libertad, por lo general, cuentan con menos recursos económicos, laborales y educativos y, además, más internas que internos mantienen sus responsabilidades familiares previas ${ }^{85}$. Esta situación ha hecho que los estudios sobre la experiencia penitenciaria femenina se hayan concentrado en encontrar el carácter especial del sufrimiento femenino dentro de las prisiones. Así, por ejemplo, se asegura que la diferencia entre la experiencia penitenciaria de hombres y mujeres se presenta en la manera en que definen, sienten y manejan el sufrimiento penitenciario ${ }^{86}$. Dicho sufrimiento estaría moldeado por la construcción del universo simbólico penitenciario, el cual, a su vez, no sería independiente del sistema de actitudes, roles y estatus otorgados a hombres y mujeres en el mundo libre ${ }^{87}$.

En este sentido, Owen ${ }^{88}$ define tres tipos de relaciones clave que moldean el universo simbólico de las prisiones de mujeres, y que ayudarían a entender tanto el contenido del sufrimiento penitenciario como los factores que lo mitigan al interior de los muros. En primer lugar, las relaciones con los hijos y la familia que se encuentran en el mundo libre; en segundo lugar, las relaciones de las prisioneras entre sí y, por último, las relaciones que mantienen con el personal administrativo y de guardia y custodia del establecimiento. Así, sostiene, las diferencias de género se manifestarían en la intensidad de las relaciones que intentan mantener con el mundo exterior y que, a su vez, se reflejarían en el mundo penitenciario a través de la construcción de relaciones más cercanas, emotivas y solidarias entre las internas y entre estas y el personal administrativo.

Las narrativas de la experiencia penitenciaria seguirían la gramática del mundo libre, en el cual la mujer es catalogada como madre y esposa. Dentro de los muros la mujer no puede escapar de su feminidad impuesta. La transformación de las relaciones familiares de las personas privadas de la libertad juega un papel clave para entender el carácter diferencial del sufrimiento penitenciario femenino. Dichas relaciones son definidas a través de un conjunto de narrativas - la interrupción, transformación y precariedad- que, a su vez, muestran distintos tipos de relaciones familiares previas al castigo penitenciario que

\footnotetext{
${ }^{85}$ ALMEDA, "Women's imprisonment in Spain", cit. nota $\mathrm{n}^{\circ} 81$.

${ }^{86}$ Ver GIALLOMBARDO, Rose, Society of women: A study of a women's prison, Chichester: Wiley, 1966.

${ }^{87}$ ROWE, Abigail, "Narratives of self and identity in women's prisons: Stigma and the Struggle for selfdefinition in penal regimes", Punishment \& Society, Vol. 13, N 5 (2011), pp. 571-591.

${ }^{88}$ OWEN, In the mix, cit. nota ${ }^{\circ} 83$, p. 119.
} 
ARIZA, Libardo; ITURRALDE, Manuel. "Mujer, crimen y castigo penitenciario".

diferencian la manera en que las mujeres asumen la transformación de sus relaciones familiares ${ }^{89}$.

De esta manera, los estudios que intentan hacer inteligible el carácter diferencial del sufrimiento penitenciario femenino, a través de los discursos de las mujeres privadas de la libertad, se encuentran con que éste se vincula estrechamente con la definición que de sí misma hace la mujer en términos de la maternidad y su posición en la estructura familiar que queda atrás. Así, la maternalización de la experiencia penitenciaria parece ineludible. La maternidad sería, en este sentido, el lenguaje con el que se habla de la cárcel femenina. Por ello, se afirma que la pérdida del rol materno es un dolor penitenciario especialmente sentido por las mujeres internas ${ }^{90}$, y en parte permite entender el interés por la suerte de los hijos e hijas de madres encarceladas ${ }^{91}{ }_{-}^{92}$, así como el énfasis en el manejo de la maternidad desde la cárcel $^{93}$, y el impacto en la estructura familiar del encarcelamiento femenino como factor explicativo de la participación posterior de los menores en actividades criminales ${ }^{94}$.

Sin restarle importancia en lo absoluto al sufrimiento penitenciario experimentado por las mujeres presas entendido como sufrimiento materno y familiar, es necesario señalar el carácter problemático de un excesivo énfasis en estos aspectos. Por una parte, por el hecho simple de que no todas las mujeres privadas de la libertad son madres o mantienen vínculos familiares estables con el mundo exterior. Identificar la experiencia penitenciaria femenina con la experiencia penitenciara de la mujer privada de la libertad que es madre reduce la complejidad del asunto, impidiendo comprender el carácter diferencial del encierro femenino más allá de lo materno. En segundo lugar, porque tiende a idealizar el discurso de la maternidad como el lenguaje clave para entender la experiencia de la mujer en cárcel, perdiendo de vista los usos de este recurso y sus efectos sobre la comprensión general del fenómeno del encierro femenino. En este sentido, Enos ${ }^{95}$ insiste en que es indispensable distinguir, en el campo penitenciario, las distintas formas en que aparece el discurso de la maternidad. Así, desde el punto vista identitario, la maternidad es una cuestión importante que da forma a un reclamo que reivindica el reconocimiento de la condición de ser madre. Este reclamo es distinto aunque estrechamente relacionado con la cuestión de cómo se ejerce la maternidad desde la prisión desde un punto de vista descriptivo y qué efectos tiene sobre el núcleo familiar. Por último, entendiendo la maternidad como un discurso político

\footnotetext{
${ }^{89}$ CHRISTIAN, Johnna; KENNEDY, Leslie, "Secondary narratives in the aftermath of crime: Defining family members' relationships with prisoners", Punishment \& Society Vol. 13, N 4 (2011), pp. 379-402.

${ }^{90}$ WARD, David; KASSEBAUM, Gene, Women's Prison. Sex and Social Structure, New Jersey: Rutgers, 2009.

91 TRAVIS, Jeremy; WAUL, Michelle, Prisoners Once Removed: The impact of incarceration and Reentry on Children, Families and Communities, Washington: The Urban Institute Press, 2003.

92 GABEL, Katherine; JOHNSTON, Denise, Children of Incarcerated Parents, New York: Lexington Books, 1995.

${ }^{93}$ Ver ENOS, Sandra, Mothering from the inside: Parenting in a women's prison, New York: State University of New York Press, 2001.

${ }^{94}$ En todo caso, el riesgo de encarcelamiento para los menores cuyas madres se encuentran en prisión es uno de los muchos que deben enfrentar, por lo que no sería preciso asumir que sus dificultades sean el resultado de la privación de la libertad de la mujer, dado que la mayoría de las familias afectadas por el encierro de la madre ya se encontraban en riesgo antes de esta circunstancia (MYERS, Barbara, et al, "Children of Incarcerated Mothers", Journal of Child and Family Studies, Vol. 8 N 1 (1999), p. 12.

${ }^{95}$ ENOS, Mothering from the inside, cit. nota $\mathrm{n}^{\circ} 93$.
} 
Polít. crim. Vol. 12, № 24 (Diciembre 2017), Art. 3, pp. 731-753.

[http://www.politicacriminal.cl/Vol_12/n_24/Vol12N24A3.pdf]

que se moviliza para lograr cambios en las condiciones de reclusión y en el régimen penitenciario. Las sensibilidades sociales que se despiertan cuando se apela a la figura de la madre que sufre por sus hijos abandonados y desvalidos en el mundo exterior, puede mitigar la demanda social punitiva y sentar las bases para la negociación de reformas institucionales penitenciarias de otro modo difíciles de plantear. 
ARIZA, Libardo; ITURRALDE, Manuel. "Mujer, crimen y castigo penitenciario".

\section{BIBLIOGRAFÍA}

ADAMS, Kenneth, “Adjusting to Prison Life”, en: TONRY, Michael (Ed.), Crime and Justice: A Review of Research, Chicago: University of Chicago Press, 1992, pp. 275359.

ALMEDA, Elizabet, "Women's imprisonment in Spain", Punishment and Society, Vol. 7, $\mathrm{N}^{\circ} 2$ (2005), pp. 183-199.

ARIZA, Libardo, "Dados sin números: un acercamiento al orden social en la cárcel La Modelo", Revista de Derecho Público, № 26 (2011), pp. 1-21.

ARIZA, Libardo; ITURRALDE, Manuel, "En contra de los pobres: justicia penal y prisiones en América Latina. El caso colombiano", en: GARGARELLA, Roberto (Ed.), El castigo penal en sociedades desiguales, Buenos Aires: Miño y Dávila, 2012.

BARBERO, Iker, , Las transformaciones del Estado y del Derecho ante el control de la inmigración, Bilbao: Ikuspegi, 2010.

BRICEÑO, Marcela, Mujeres y prisión en Colombia: Análisis desde una perspectiva de Derechos Humanos y Género, Bogotá: Procuraduría Delegada en lo Preventivo para Derechos Humanos y Asuntos Étnicos-Grupo de Asuntos Penitenciarios y Carcelarios, 2006, p. 30.

BRITTON, Dana, "Feminism in Criminology: Engendering the Outlaw", Annals of the American Academy of Political and Social Science, Vol. 571, $\mathrm{N}^{\circ} 1$ (2000), pp. 57-76.

CAIN, Maureen, "Feminists transgress criminology", Introducción en CAIN, Maureen (Ed.), Growing Up Good: policing the behavior of girls in Europe, London: Sage, 1989.

CARLEN, Pat, Sledgehammer: Women's Imprisonment at the Millennium, London: Palgrave Macmillan, 1998. , Criminal Women, Cambridge: Polity Press, 1995.

, Criminal women and criminal justice: The limits to, and potential of, left realist perspectives, London: Sage, 1992.

, Alternatives to Women's Imprisonment, Oxford: Open University Press, 1990, 137, p. 114.

, Women's Imprisonment: A Study in Social Control, London: Routledge \& K. Paul, 1983, p. 248.

CARLEN, Pat; WORRALL, Anne, Analysing Women's Imprisonment, Cullompton: Willan Publishing, 2004, P. 61.

CARLTON, Bree; SEGRAVE, Marie, “Women's survival post-imprisonment: Connecting imprisonment with pains past and present", Punishment \& Society, Vol. 13, $\mathrm{N}^{\circ} 5$ (2011), pp. 551-570.

CARRILlO, William, "From my Prison Cell. Time and Space in Prison in Colombia, an ethnographic approach", Latin American perspectives, Vol. 28, № 116 (2001), pp. 149-164.

CENTRO DE ESTUDIOS LEGALES Y SOCIALES -CELS- et al, Mujeres en prisión. Los alcances del castigo, $1^{\mathrm{a}}$ ed., Buenos Aires: Siglo Veintiuno Editores, 2011, p. 1011.

CHRISTIAN, Johnna; KENNEDY, Leslie, "Secondary narratives in the aftermath of crime: Defining family members' relationships with prisoners", Punishment \& Society Vol. 13, N 4 (2011), pp. 379-402. 
Polít. crim. Vol. 12, No 24 (Diciembre 2017), Art. 3, pp. 731-753.

[http://www.politicacriminal.cl/Vol_12/n_24/Vol12N24A3.pdf]

COUSINS, Mark, "Men's rea: a note on sexual difference, criminology and the law", en: CARLEN, Pat; COLLISON, Mike (Eds.), Radical Issues in Criminology, New Jersey: Barnes and Noble Books, 1980.

DAUVERGNE, Catherine, Making People Illegal: What Globalization Means for Migration and Law, Cambridge: Cambridge University Press, 2008.

DE GIORGI, Alessandro, "Immigration control, post-Fordism, and less eligibility: A materialist critique of the criminalization of immigration across Europe", Punishment \& Society, Vol. 12, N² (2010), pp. 147-167.

DE ROSIA, Victoria, Living inside prison walls: adjustment behavior, Connecticut: Praeger, 1998,

DENNIS. Norman; ERDOS, George, Families Without Fatherhood, London: IEA Health and Welfare Unit, 1992.

DEVLIN, Angela, DEVLIN, A, Invisible Women. What's Wrong with Women's Prisons?, Winchester: Waterside Press, 1998.

DOBASH, Russell; DOBASH, R. Emerson; GUTTERIDGE, Sue, The Imprisonment of Women, Oxford: Blackwell, 1986.

EATON, Mary, Justice for Women? Familiy, Court and Social Control, Buckingham: Open University press, 1986.

EDWARDS, Susan, Women on Trial. A Study of the Female Suspect, Defendant and Offender in the Criminal Law and Criminal Justice System, Manchester: Manchester University Press, 1984.

ENOS, Sandra, Mothering from the inside: Parenting in a women's prison, New York: State University of New York Press, 2001.

FEELEY, Malcolm; LITTLE, Deborah, "The vanishing female: The decline of women in the criminal process", 1687-1912", Law and Society Review, Vol. 25, № 4 (1991), pp. 719-757.

FINKELSTEIN, Ellis, Prison Culture: An Inside View, Averbury: Aldershot, 1993.

GABEL, Katherine; JOHNSTON, Denise, Children of Incarcerated Parents, New York: Lexington Books, 1995.

GIALLOMBARDO, Rose, Society of women: A study of a women's prison, Chichester: Wiley, 1966.

GOFFMAN, Irvin, Asylums: Essays on the social situation of mental patients and other inmates, London: Penguin Books, 1961.

HAGAN, John; SIMPSON, John; GILLIS, A.R, "The Sexual Stratification of Social Control: A Gender-Based Perspective on Crime and Delinquency", The British Journal of Sociology, Vol. 30, № 1 (1979), pp. 25-38.

"Feminist Scholarship, Relational and Instrumental Control, and a PowerControl Theory of Gender and Delinquency", The British Journal of Sociology, Vol. 39, $\mathrm{N}^{\circ} 3$ (1988), pp. 301-336.

INSTITUTO NACIONAL PENITENCIARIO Y CARCELARIO, 2013, Estadísticas oficiales, disponibles en: http://www.inpec.gov.co/portal/page/portal/Inpec/Institucion/Estad\%EDsticas/Estadis ticas/Estad\%EDsticas

ITURRALDE, Manuel, La revolución desde arriba: la sociología política del neoliberalismo de Wacquant y su relevancia para América Latina, Bogotá: Siglo del 
ARIZA, Libardo; ITURRALDE, Manuel. "Mujer, crimen y castigo penitenciario".

Hombre, Universidad de los Andes, Pontificia Universidad Javeriana-Instituto Pensar, 2013.

"Prisiones y castigo en Colombia: la construcción de un orden social excluyente", en: ARIZA, Libardo; ITURRALDE, Manuel, Los muros de la infamia. Prisiones en Colombia y América Latina, Bogotá: Colección CIJUS-Uniandes, 2011. , Castigo, liberalismo autoritario y justicia penal de excepción, Bogotá: Siglo del Hombre, Universidad de los Andes, Pontificia Universidad Javeriana-Instituto Pensar, 2010.

"Democracies without Citizenship: Crime and Punishment in Latin America", New Criminal Law Review, Vol. 13, N 2 (2010), pp. 309-332.

KENNEDY, Helena, Eve was Framed: Women and British Justice, London: Chatto \& Windus, 1992.

LOMBROSO, Cesar; FERRERO, William, The Female Offender, New York: D. Appleton and Company, 1898, p. 28.

MELOSSI, Dario, "In a peaceful life: Migration and the crime of modernity in Europe", Punishment \& Society, Vol. 5, N 4 (2003), pp. 371-397.

MURRAY, Charles, The Emerging British Underclass, London: Institute of Economic Affairs, 1994.

MYERS, Barbara, et al, "Children of Incarcerated Mothers", Journal of Child and Family Studies, Vol. $8 \mathrm{~N}^{\circ} 1$ (1999), p. 12.

NORZA-CÉSPEDES, Ervyn; GONZÁLEZ-ROJAS, Andrea; MOSCOSO-ROJAS, Manuel; GONZÁLEZ-RAMÍREZ, Juan David, "Descripción de la criminalidad femenina en Colombia: factores de riesgo y motivación criminal", Revista Criminalidad, Vol. 58, $\mathrm{N}^{\circ} 1$ (2012), pp. 339-357.

OWEN, Barbara, In the mix: Struggle and Survival in a Women's prison, Albany: State University of New York Press, 1998.

PRATT, John, Penal Populism, London: Routledge, 2007.

ROWE, Abigail, "Narratives of self and identity in women's prisons: Stigma and the Struggle for self-definition in penal regimes", Punishment \& Society, Vol. 13, N 5 (2011), pp. 571-591.

SMART, Carol, "The New Female Criminal: Reality or Myth?", British Journal of Criminology, Vol. 19, $\mathrm{N}^{\circ} 1$ (1979), pp. 50-59.

, "Law, Crime and Sexuality", Essays on Feminism, Vol. 1, London: Sage Publications, 1995.

,"Feminist approaches to Criminology or Post modern woman meets atavistic man", en: GELSTHORPE, Lorraine; MORRIS, Allison (Eds.), Feminist Perspectives in Criminology, Buckingham: Open University Press, 1990.

SMITH, Ann, Women in Prison: A Study in Penal Methods, London: Stevens, 1962,

SUDBURY, Julia, Global lockdown: Race, Gender, and the Prison Industrial Complex, New York: Routledge, 2005.

SYKES, Gresham M., The Society of Captives, Princeton: Princeton University Press, 1958.

TRAVIS, Jeremy; WAUL, Michelle, Prisoners Once Removed: The impact of incarceration and Reentry on Children, Families and Communities, Washington: The Urban Institute Press, 2003.

THOMAS, W. I., "The Adventitious Character of Woman", American Journal of Sociology, Vol. 12, $\mathrm{N}^{\circ} 1(1906)$, pp. 32-44. 
Polít. crim. Vol. 12, No 24 (Diciembre 2017), Art. 3, pp. 731-753.

[http://www.politicacriminal.cl/Vol_12/n_24/Vol12N24A3.pdf]

VAN GUNDY, Alana; BAUMANN-GRAU, Amy, Women, Incarceration, and Human Rights Violations: Feminist Criminology and Corrections, London: Ashgate, 2014.

WACQUANT, Löicq, "Suitable enemies: Foreigners and immigrants in the prisons of Europe", Punishment \& Society, Vol. 1, № 2 (1999), pp. 215-222.

WALMSLEY, Roy, International Center for Prison Studies: World Female Imprisonment List. $3^{\text {rd }}$ ed., London: World Prison Brief, 2015, p. 2.

WARD, David; KASSEBAUM, Gene, Women's Prison. Sex and Social Structure, New Jersey: Rutgers, 2009.

WORRALL, Anne, Offending women: Female Lawbreakers and the Criminal Justice System, London: Routledge, 1990.

, "Out of place: Female offenders in court", Probation Journal, Vol. 28, $\mathrm{N}^{\circ} 3$ (1981), pp. 90-93. 